\title{
Optimizing a multi-channel ANC system for broadband noise cancellation in a telephone kiosk using genetic algorithms
}

\author{
Allahyar Montazeri* and Javad Poshtan \\ Faculty of Electrical Engineering, Iran University of Science and Technology, Narmak 16846, Tehran, Iran
}

Received 4 July 2007

Revised 2008

\begin{abstract}
The problem of optimizing an active noise control system for the implementation of a multi-channel ANC system with the aim of global reduction of broadband noise in a telephone kiosk is addressed in this paper. This optimization involves finding best locations for loudspeakers and microphones, and optimizing control signals. The problem of finding a proper size for control system, i.e. the number of loudspeakers and microphones involved in the control system is also investigated. The mean of acoustic potential energy in the enclosure in a frequency range of $50 \mathrm{~Hz}$ to $300 \mathrm{~Hz}$ is selected as a measure for optimization. Several genetic algorithms are proposed and compared to find the global minimum of this performance index. In order to have a better performance in reaching the global minimum, the parameters of these genetic algorithms are tuned, and the best genetic algorithm is selected among them. Numerical simulations of the acoustical potential energy and also sound pressure at the height where the head of a person may be located, confirms the optimality of the locations proposed by the genetic algorithm. Besides, the robustness of the optimized control system with respect to eventual changes in the location of primary and secondary loudspeakers and also microphones is shown with several simulations.
\end{abstract}

Keywords: Genetic algorithms, active noise control, optimal placement, global sound reduction

\section{Introduction}

Active control of sound and vibration is not just a signal processing problem, and the achievable level of control is dependent on some additional parameters that are unrelated to the signal processing aspects of the problem [1]. Once the cost function that must be minimized (such as sound power, sound pressure at a number of locations, acoustic potential energy...) has been chosen to achieve an overall optimized system, its parameters must be optimized in a strict sequence [7]. This procedure consists of the optimization of two major subsystems: physical and electronic subsystems. Optimization of the electronic subsystem deals mainly with control and signal processing problems, where the controller must be designed to meet the control objectives. On the other hand, the optimization of physical subsystem is to determine the number and positions of control sources and error sensors [7]. In fact arrangement of control sources (their number and locations) determines the maximum achievable reduction of the performance index, when error sensors and electronic controller are also optimized. Arrangement of error sensors (their number and locations) in the next step is of great importance in the sense that they must be able to approximate the performance index (acoustic potential energy here) as close as possible to attain the maximum level of noise reduction in the enclosure. Besides, it is worth to notice that the place of microphones have mutual effect in the optimized loudspeaker positions, and hence it is wise to optimize them together.

\footnotetext{
${ }^{*}$ Corresponding author. E-mail: amontazeri@iust.ac.ir.
} 
Multiple regression is one of the methods used for the optimal placement of microphones and loudspeakers in an enclosure [14]. In this method, considering the analytical description of the acoustical behavior of an enclosure, a shortcut is proposed for the placement of sensors and actuators individually. Consequently one can compare the effect of various placements of loudspeakers and microphones. In general, the placement of sensors and actuators is based on numerical search methods. Gradient methods generally are not efficient tools for the optimization of sensor and actuator configurations in an ANC system. This is due to the nature of the problem that allows the calculation of the effect of the ANC system only when the sensor and actuator configurations are specified. Hence, finding a well-behaved performance index as a function of the positions of sensors and actuators for calculation of gradients is not a straightforward task. Besides, the complex multi-modal nature of most practical active sound and vibration control applications involving large structures and multiple control sources in nature make gradient methods ineffective for searching global optimum arrangement.

One of the solutions for such optimization problems relies on the so-called natural algorithms. These algorithms may be divided in three general categories: genetic algorithms [6], simulated annealing [15], and recently proposed swarm intelligence [16]. The applications of using these kinds of natural algorithms in optimization of active noise and vibration control systems are reported for example in $[1,17]$.

Ruckman and Fuller [13] examined the problem of optimal placement of only actuators using subset selections. The use of genetic algorithms for optimizing just the error sensors is also reported in [10]. A two step approach for optimizing the positions of loudspeaker and microphones for active control of the sound radiated by electrical transformer is adopted in [11]. They first determined the configuration of actuators based on the model of primary field, then a genetic algorithm was used to determine the error sensor configuration. Nevertheless, an additional difficulty in this problem is that sensor/actuator configurations cannot be optimized independently, since the effect of the ANC system directly depends on the combined sensor and actuator configuration. Different nonlinear optimization techniques are used and compared in [3], including genetic algorithms, for simultaneous optimization of the positions of loudspeakers and microphones in a double-panel partition. They have concluded that gradient methods converge faster and yield better final solution for this application. The application of genetic algorithms for optimal placement of sensors and actuators inside an aircraft cabin is studied in [4]. Their proposed genetic algorithm uses integer coding and it is repeated for different initial population sizes to achieve the best results.

Phones are currently common devices used for communications. However, in many places, such as a noisy street or an industrial place, the communication suffers from excessive noise. A common way to reduce such environmental noise is to place the phone set in an enclosure; However, since the main components of street or industrial noise cover frequencies from below $100 \mathrm{~Hz}$ to over $500 \mathrm{~Hz}$, active noise control (ANC) systems are a potential solution to improve the quality of communication in telephone kiosks by reducing the noise inside the cabin. The analysis and feasibility study for global reduction of broandband noise in such an application is studied by the same authors [12], and showed that due to the existence of degenerate modes the control action is complicated and needs more considerations. On the other hand since ANC systems are application specific, they should be optimally designed for each application and the most important parameters that must be optimized are positions of loudspeakers and microphones (Fig. 1).

Moreover, to achieve optimal convergence behavior, each genetic algorithm must be "tuned" to suit the particular problem at hand. In fact, reaching a near-optimum solution in a reasonable time for applications with large search spaces (like the application in hand in this paper) requires this as a necessity.

In this paper a multi-channel ANC system for global reduction of broadband noise in a telephone kiosk is optimized. For this purpose two constraint genetic algorithms with different coding schemes are proposed. These algorithms are carefully tuned and compared with each other and also with the random search method, in terms of the quality of the obtained solution and convergence time. In next section the model of the kiosk is described and also the problem to be solved by genetic algorithm is stated. In Section 3, considering practical limitation for implementation of the ANC system, the proposed genetic algorithms are introduced and compared. Based on the obtained optimal position of microphones and loudspeakers in Section 4, the performance of the ANC system and also its robustness with respect to possible changes in the optimal positions is studied in Section 5. 


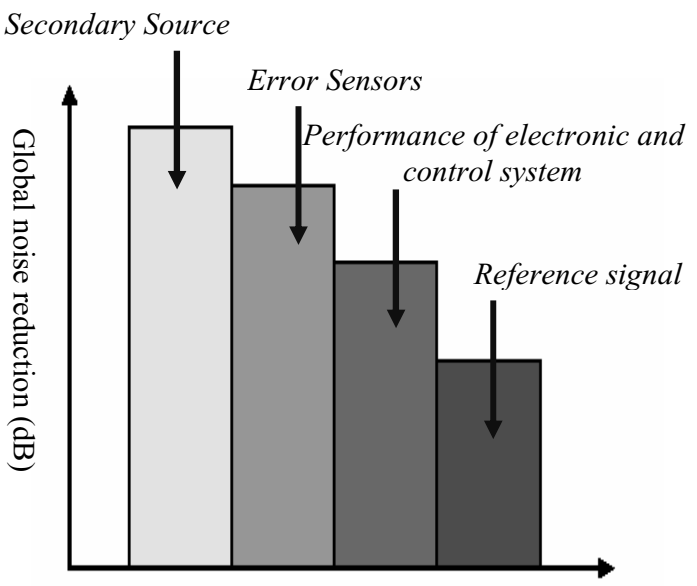

Fig. 1. Parameters affecting the optimal performance of an ANC system. (ranked by importance [8]).

\section{Problem statement and modeling of the kiosk}

The experimental kiosk aimed for implementation of the ANC system, and its model is shown in Fig. 2. Suppose we would like to minimize the acoustical pressure produced by a speaker (primary source) in an enclosure as shown in Fig. 2. The speaker emits colored noise in the frequency range from $50 \mathrm{~Hz}$ to $300 \mathrm{~Hz}$, and we intend to minimize the mean of the acoustic potential energy in this frequency range. A thorough modal analysis of this enclosure was performed in a separate work [12] in which the range of the frequency for which global reduction of broadband noise is possible has been determined. It has also shown that the position of loudspeakers and microphones has substantial effect on the maximum achievable reduction of acoustic potential energy, and the corner of the enclosure will not be a good choice for positioning the loudspeakers because of existence of degenerate modes. In this section the aim is to come up with a brief description of modeling acoustical environment of the enclosure required for optimization with genetic algorithm. Also some computational aspects of this optimization problem are discussed.

The governing wave equation in a three dimensional environment, taking into account the viscous damping of environment, and assuming a sound source with specific volume velocity distribution in right hand side, may be expressed as

$$
\frac{1}{c_{0}^{2}} \frac{\partial^{2}}{\partial t^{2}} p(\mathbf{x}, t)-\mu \nabla^{2} \frac{\partial}{\partial t} p(\mathbf{x}, t)-\nabla^{2} p(\mathbf{x}, t)=\rho_{0} \frac{\partial}{\partial t} g(\mathbf{x}, t),
$$

where $c_{0}$ is the speed of sound in enclosure, $p(\mathbf{x}, t)$ is the acoustical pressure at point $\mathbf{x}$ and time $t, \rho_{0}$ is the density of air, $\mu$ (with the dimension of $s^{-1}$ ) is a constant indicating damping in air or walls, and $g(\mathbf{x}, t)$ is volume velocity of sound per volume. Since the acoustical pressure $p$ in comparison with atmosphere pressure is very small, the linearity of Eq. (1) is assured. In addition to Eq. (1), there are six boundary conditions assuming the walls of enclosure are rigid. As the velocity on walls is zero, acoustical pressure will not transmit outside and hence pressure gradient on walls will become zero. As a result, the boundary conditions of Eq. (1) will become

$$
\begin{array}{ll}
p_{x}(x, y, z, t)=0 & x=0, L_{1} \\
p_{y}(x, y, z, t)=0 & y=0, L_{2} \\
p_{z}(x, y, z, t)=0 & z=0, L_{3} .
\end{array}
$$

The solution of Eq. (1) with boundary conditions as in Eq. (2) are standing waves in steady state which can be expressed in terms of some basis functions called the acoustical modes of enclosure. Assuming a harmonic excitation in right hand-side of Eq. (1), the amplitude of complex pressure at a specific point and frequency can be obtained as

$$
p(\mathbf{x}, \omega)=\sum_{n=1}^{\infty} \psi_{n}(\mathbf{x}) a_{n}(\omega)=\psi^{T} \mathbf{a} .
$$




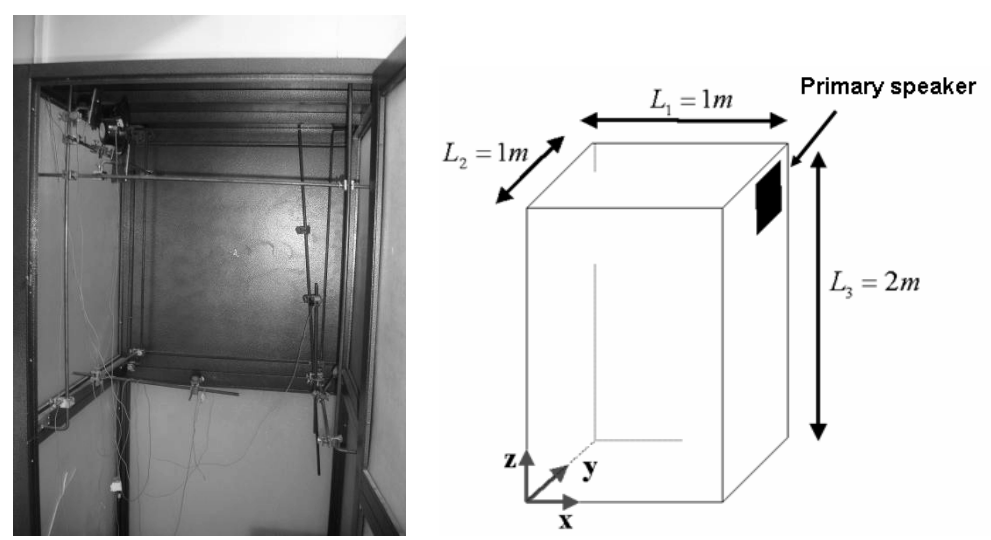

Fig. 2. Experimental kiosk and its model with primary source and assigned coordinate system.

In Eq. (3), $\psi_{n}(\mathbf{x})$ is the $n$th mode shape of the enclosure, and $a_{n}(\omega)$ is its amplitude [2] and Appendix A. By truncation of Eq. (3) to the first $\mathrm{N}$ modes, $\psi$ and a becomes $N \times 1$ vector of these values. In order to have a good approximation of acoustical pressure in the enclosure, it is necessary to include as many as possible modes in the computation of Eq. (3). However, because of reducing computational time mainly for genetic algorithm, a trade-off is required. This problem is addressed in next section. As stated by Eq. (3), the optimization procedure relies on modeling the noise inside kiosk as modal series including constant damping. In acoustics, modal series usually do not match experimental data closely. This is mainly due to the fact that the acoustical behavior of a real kiosk is much more complex than the model calculated by Eq. (1): walls are not infinitely stiff, and there are windows, doors, openings, etc. In addition, it is the polar pattern and the acoustic source impedance of the given loudspeaker that determines which of the potential room modes are actually excited and to which degree. This also will cause damping of modes vary dramatically in the interested frequency range. However, the theoretical study in the paper provides the necessary tools required for a real case in practice.

A suitable starting point for control of the acoustical field in an enclosed cavity with rigid walls is the active control of sound at a few distinct points in the enclosure. However, minimization of the acoustical field at distinct points in a cavity does not necessarily give the best result from a global-reduction viewpoint. In order to achieve the maximum level of reduction for a specified configuration of microphones and loudspeakers, the problem must be formulated based on a more global error criterion. In this regard, reduction of acoustic potential energy is a suitable global performance criterion for the acoustic field in an enclosure.

The acoustic potential energy in an enclosure may be obtained from

$$
E_{p}=\frac{1}{4 \rho_{0} c_{0}^{2}} \int_{V}|p(\mathbf{x}, \omega)|^{2} d V,
$$

where the acoustical pressure in an enclosure is computed by Eq. (3). According to Eqs (1) and (2), acoustic potential energy in an enclosure can be expressed in terms of mode amplitudes as

$$
E_{P}=\frac{V}{4 \rho_{0} c_{0}^{2}} \mathbf{a}^{H} \mathbf{a} .
$$

Considering the linear behavior of acoustic fields yielding the principle of superposition, the amplitude of each mode at a certain frequency can be expressed as:

$$
\mathbf{a}=\mathbf{a}_{p}+\mathbf{B q}_{s}
$$

where $\mathbf{a}_{P}$ shows the effect of the primary source on the amplitude of acoustic modes, is a matrix whose element $B_{n m}$ shows the effect of the $m$ th source on the $n$th mode, and $\mathbf{q}_{s}$ is the vector of volume velocities of secondary sources [2] and Appendix A.

By substituting Eq. (6) in Eq. (5), the potential energy in the enclosure is obtained directly in terms of control inputs 


$$
E_{P}=\frac{V}{4 \rho_{0} c_{0}^{2}}\left(\mathbf{q}_{s}^{H} \mathbf{B}^{H} \mathbf{B} \mathbf{q}_{s}+\mathbf{q}_{s}^{H} \mathbf{B}^{H} \mathbf{a}_{P}+\mathbf{a}_{P}^{H} \mathbf{B} \mathbf{q}_{s}+\mathbf{a}_{p}^{H} \mathbf{a}_{P}\right) .
$$

It is evident from Eq. (7) that the potential energy in an enclosure is a quadratic function of the volume velocity of secondary sources, and hence the optimum vector that minimizes this expression will be

$$
\mathbf{q}_{\text {so }}=-\left[\mathbf{B}^{H} \mathbf{B}\right]^{-1} \mathbf{B}^{H} \mathbf{a}_{P} \text {. }
$$

By substituting Eq. (8) in Eq. (7), the minimum potential energy will be obtained as

$$
E_{\text {Po }}=\frac{V}{4 \rho_{0} c_{0}^{2}}\left(\mathbf{a}_{P}^{H} \mathbf{a}_{P}-\mathbf{a}_{p}^{H} \mathbf{B}\left[\mathbf{B}^{H} \mathbf{B}\right]^{-1} \mathbf{B}^{H} \mathbf{a}_{P}\right) .
$$

A more practical solution from control system perspective, where tuning the amplitude and phase of control sources must be done automatically, acoustic potential energy in enclosure is approximated by measurement of acoustic pressure at some discrete points using microphones. In this case, the performance index is the sum of square amplitude of acoustic pressure at selected points, and its minimization will result in the optimum volume velocity of control sources. The approximation of acoustic potential energy in Eq. (4) is

$$
J_{P}=\frac{V}{4 \rho_{0} c_{0}^{2} M} \mathbf{p}^{H} \mathbf{p},
$$

where $\mathbf{p}$ is the vector of acoustic pressure at $M$ discrete point, and can be written by the principle of superposition as (Appendix A)

$$
\mathbf{p}=\mathbf{p}_{p}+\mathbf{Z q}_{s} .
$$

Here, $\mathbf{p}_{p}$ and $\mathbf{Z}$, unlike $\mathbf{a}_{p}$ and $\mathbf{B}$ are measurable quantities and can be used for practical implementation of ANC system. By combination of Eqs (10) and (11) the performance index is computed directly in terms of the volume velocity of secondary sources as follows

$$
J_{p}=\frac{V}{4 \rho_{0} c_{0}^{2} M}\left[\mathbf{q}_{s}^{H} \mathbf{Z}^{H} \mathbf{Z} \mathbf{q}_{s}+\mathbf{q}_{s}^{H} \mathbf{Z}^{H} \mathbf{p}_{p}+\mathbf{p}_{p}^{H} \mathbf{Z} \mathbf{q}_{s}+\mathbf{p}_{p}^{H} \mathbf{p}_{p}\right] .
$$

Minimization of this quadratic function will give the optimal value and the minimum of performance index as

$$
\begin{aligned}
& \mathbf{q}_{\text {so }}=-\left[\mathbf{Z}^{H} \mathbf{Z}\right]^{-1} \mathbf{Z}^{H} \mathbf{p}_{p} \\
& J_{\text {po }}=\frac{V}{4 \rho_{0} c_{0}^{2} M}\left(\mathbf{p}_{p}^{H} \mathbf{p}_{p}-\mathbf{p}_{p}^{H} \mathbf{Z}\left[\mathbf{Z}^{H} \mathbf{Z}\right]^{-1} \mathbf{Z}^{H} \mathbf{p}_{P}\right) .
\end{aligned}
$$

Here for optimization of the multi-channel ANC system, the volume velocity of control source is calculated from Eq. (13), but since we intend to minimize acoustical potential energy in the enclosure this control signal is replaced in Eq. (7), and optimization is performed for possible positions of loudspeaker and microphones. Although in the formulation above, the responses of the kiosk is calculated by the sum of the response of several distinct modes to the excitation signal, this model can be evaluated at each frequency, and hence provides a mean for studying optimization procedure over the interested frequency range or the frequency range of the broadband incident disturbance.

The optimization of positions of loudspeakers and microphones is a continuous-domain problem. The loudspeakers or microphones must be moved in two or three dimensional space, and the performance index which is the maximum acoustic potential energy reduction of enclosure must be calculated. To show a typical performance index of such optimization problems an example is shown in Fig. 3. Two primary speakers Sp1 and Sp2 are placed at two opposite walls of the enclosure and work at the frequency of $255 \mathrm{~Hz}$. It is assumed that the secondary source S4 is fixed and $\mathrm{S} 2$ is moving in $y$ and $z$ direction in the plane as shown in Fig. 3. For each position of S2, its optimum volume velocity (the amplitude and phase according to Eq. (13) is calculated, and the maximum acoustical potential energy reduction is calculated. Figure 4 shows this quantity as a function of positions of S2 in the specified plane.

The shape of the resulted curve in Fig. 4 shows the main characteristics of a performance index which must be optimized for a practical ANC system. This multi modal nature of most applications in active control of sound and vibrations, especially in cases with large dimensions and many error sensors and control actuators, causes many difficulties when using gradient algorithms. In the problem shown in Fig. 3 based on the initial position of the 


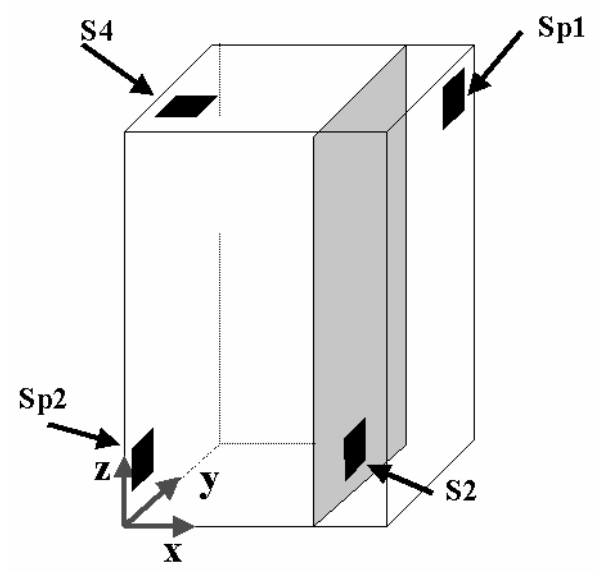

Fig. 3. Telephone kiosk with two primary and secondary sources where S2 is moving in the specified plane in $y$ and $z$ direction.

secondary source, any gradient algorithms used for optimization may converge to any local maxima or the global maximum of this performance index. In fact, the variations of performance index by movement of one of the secondary sources will be dependent to the position of other secondary sources. Although it is possible to start gradient algorithms from different initial conditions, there is always a risk that the obtained solution correspond to a sharp peak of performance surface and thus be very sensitive to small changes in the position of secondary sources or microphones [5].

If natural algorithms are applied to continuous domain problem like the one shown in Fig. 3, the position of secondary sources and microphones must be restricted to several specific positions and hence the problem will be changed to a combinatorial problem. Before doing any optimization, based on practical and economical limitations it is necessary to determine the possible positions for installation of loudspeakers and microphones. In the problem at hand for the obtained solutions the following practical consideration is included in the coded genetic algorithms:

- The loudspeakers and microphones are not allowed to occupy the same positions for any obtained solution.

- Since the loudspeakers are considered as a distributed source with the dimension of $15 \mathrm{~cm}$, this point is taken into account in discretizing the search space.

- Since placing the secondary loudspeakers very close to the primary speaker will be trivial (and in many situations impractical) solution the secondary loudspeakers are not allowed to be positioned in a cubic space with the center of primary speaker and dimensions of $30 \mathrm{~cm}$.

- Because of limitations of the available control channels (channels of DA card) for now the number of loudspeaker is determined two.

Regarding the above limitations, the width, length and height of the enclosure are divided into 16 segments, and hence the search space for possible locations of loudspeakers and microphones will have 4096 points totally. Since the aim is the global reduction of noise in a kiosk, the number of microphones required to approximate the potential energy of the enclosure must be large.

A rationale proportion for the number of microphones to loudspeakers pointed in literature is 2 to 1 [9]. To observe this ratio, 4 microphones are included in optimization routine. With this number of microphones and loudspeakers the problem faced is to select 6 positions (two for loudspeakers and four for microphones) among 4096 positions, and to examine which configuration of control system will result in the maximum acoustic potential energy reduction in the enclosure. This is equal to searching $C_{6}^{4096}=\frac{4096 !}{6 ! 4090 !} \approx 6.5 \times 10^{18}$ different points, and if the calculation of potential energy for each solution takes about $1 \mathrm{msec}$ by nowadays computers, the examination of all possible configurations for error sensors and control actuators will take a time around $20.61 \times 10^{8}$ years. So the use of a guided random search algorithm like genetic algorithm will be a good choice to solve such a big combinatorial problem. 


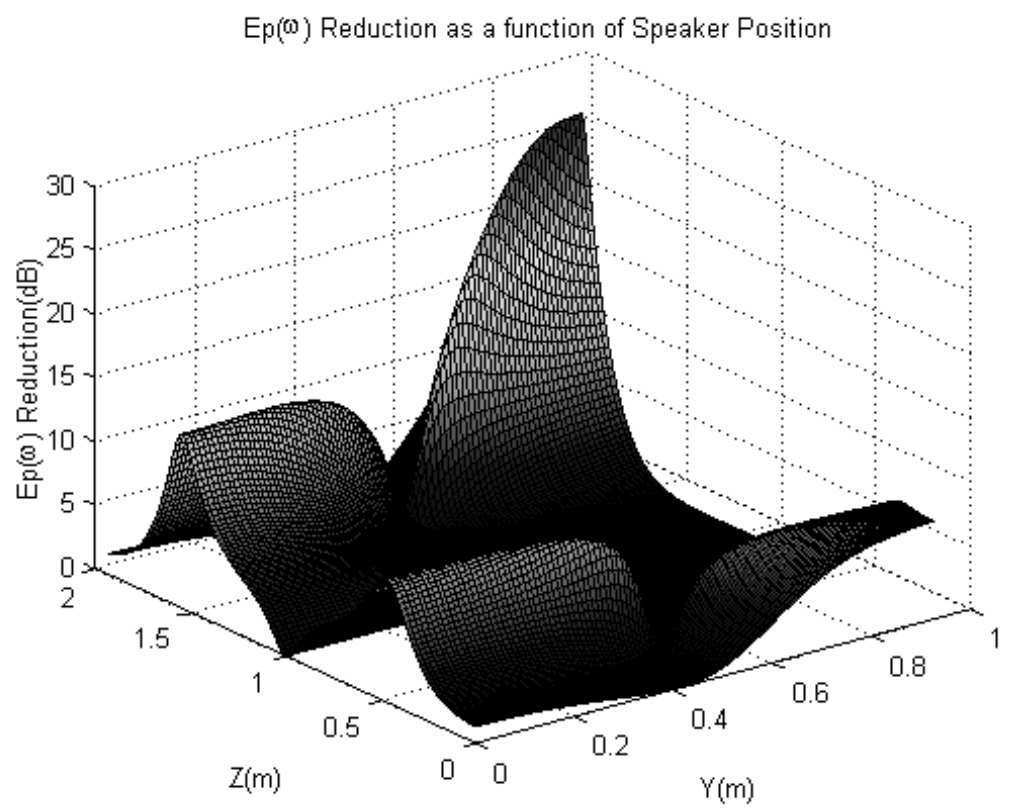

Fig. 4. Maximum acoustic potential energy reduction in enclosure as a function of the position of S2 when two primary sources work in $255 \mathrm{~Hz}$.

\section{The proposed genetic algorithm}

Genetic algorithm is a well-known guided random search used for optimization purposes. An ordinary genetic algorithm starts with an initial population, whose size may vary for different problems, and by continuously producing the next generations tries to find the optimum solution for a problem at hand. For the optimization of locations of microphones and speakers, the initial population size is usually chosen from 40 to 100 individuals [7]. Finding the optimum solution consists of three main steps: evaluating the fitness of the current population, selection of proper parents, and then evolving new children. In addition, in each generation, the fittest individual is transmitted directly to the next generation (elitism) to guarantee that we have not lost the best individual in the entire search process.

Figure 5 [6] shows a comparison among various optimization methods, describing the performance of different optimization algorithms along with diversities of problem they can handle. Genetic algorithms are known in many fields for their optimization capabilities [6] when the cost function is not directly related to optimization parameters. Here they are used for optimal placement of speakers and microphones (simultaneously) in a three dimensional enclosure for the global reduction of sound pressure.

The key factor in achieving the global optimum point is to keep a balance between the diversity of population and selective pressure to force higher fitness solutions. The control parameters in genetic algorithms are the size of the initial population, coding of problem variables, crossover probability, and mutation probability. By suitably choosing these parameters, a genetic algorithm will be tuned for a specific problem to find the global optimum point. In the following, the proposed genetic algorithm and its performance for the problem of optimizing the location of microphones and loudspeakers is discussed in more details.

\subsection{Coding scheme}

In this problem, the positions of sensors and actuators are selected as the optimization variables and described in a Cartesian coordinate system. The selection of a special type of coding scheme in a specific problem may have influential effect on the performance and convergence behavior of the algorithm in reaching the final solution. The first coding method used here is integer coding. In fact considering the segmentation discussed in Section 2, there are 4096 positions corresponding to 4096 integer numbers inside the kiosk. Thus for each position of loudspeakers 


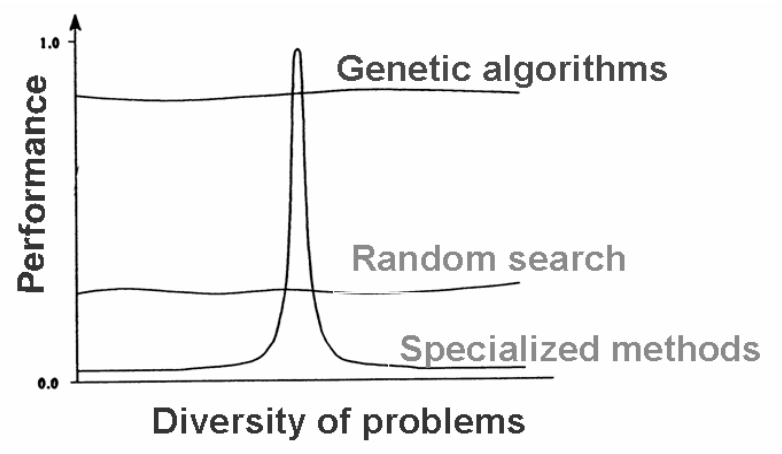

Fig. 5. A comparison among various optimization methods used in problems.

or microphones, an integer number (between 1 and 4096) determining its position is assigned. The crossover is performed probabilistically by interchanging the integer numbers of each chromosome with one another, and mutation is used to change one of the integer numbers in the chromosome by a random integer number between 1 and 4096. The main advantage of this method is its capability to use along with finite element models where each node is determined with an integer number. With this method each solution for arrangement of loudspeakers and microphones in enclosure is coded with an integer string called Chromosome ${ }_{1}$

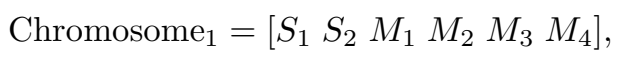

where $S_{1}$ and $S_{2}$ represent the positions of two secondary speakers, and $M_{1}$ to $M_{4}$ show the positions of four error microphones. Each of these six elements is an integer number between 1 and 4096. The second coding scheme used is multivariable binary string (MVBS). In this method any variable with upper limit $u$ and lower limit $l$ is divided into $2^{n}$ values, and to each of the obtained numbers an $n$-bit binary number will be assigned. So, for representing a number in the range of the dimensions of the kiosk with 16 divisions, a 4-bit gray code is used. The gray code prevents a sudden jumping of search variables when crossover happens, and hence allows the algorithm to search more effectively over the entire solution space. Each solution for arrangement of the microphones and speakers in the enclosure is coded with a binary string called Chromosome ${ }_{2}$

$$
\text { Chromosome }_{2}=\left[S_{1} S_{2} M_{1} M_{2} M_{3} M_{4}\right] \text {. }
$$

In this string, the same as for the integer coding, $S_{1}$ and $S_{2}$ represent the location of speakers, and $M_{1}$ to $M_{4}$ indicate the location of microphones. Each element of Chromosome ${ }_{2}$ consists of 3 binary numbers of 4-bit lengths (to show the coordinates of microphones and speakers), so each Chromosome ${ }_{2}$ will have a length of 72 bits.

\subsection{Fitness evaluation}

Performance index is the main factor by which the value of each chromosome is determined. This function accepts chromosomes as inputs, and evaluates them by generating a number. In fact performance index will establish the connection between the genetic algorithm and the optimization problem. Since the aim is global reduction of broadband noise inside the enclosure in our genetic algorithm, the average of acoustical potential energy reduction $(A P R)$ in the bandwidth between 50 to $300 \mathrm{~Hz}$ is adopted as the performance index and is defined by

$$
\begin{aligned}
& A P R\left(\omega_{i}\right)(d B)=-10 \log _{10} \frac{E_{p o}\left(\omega_{i}\right)}{E_{p p}\left(\omega_{i}\right)} \\
& \text { Average } A P R(d B)=\frac{1}{\omega_{N}-\omega_{0}} \sum_{\omega=\omega_{0}}^{\omega_{N}} A P R\left(\omega_{i}\right),
\end{aligned}
$$

where $\omega_{0}$ and $\omega_{N}$ are the minimum and maximum points of the frequency range of interest, and $E_{p o}$ and $E_{p p}$ are acoustic potential energy in the kiosk before and after using the ANC system. In the simulation of the genetic 
algorithm, calculation of performance index for each generation is the most time consuming part of the algorithm. This is actually because of the inclusion of a large number of modes required to evaluate Eqs (8) and (9). To reduce the number of modes when calculating Eq. (17), a method using Schroeder frequency of the enclosure is considered [18]. This frequency is defined as the frequency where the modes start bunching so closely together that they no longer are seen as resonant peaks, and hence their contribution to the modal response of the kiosk can be neglected. By assuming the average absorption coefficient of 0.15 for the kiosk, the calculated Schroeder frequency is $659.3 \mathrm{~Hz}$, and there are about 100 modes below this frequency. Although this number of modes may be sufficient for future computations, there are some especial positions that require larger number of modes to have more accurate results. Figure 6 shows the sound pressure level at $300 \mathrm{~Hz}$ in terms of increasing number of modes. The part highlighted by bold line shows the sound pressure level (SPL) for different positions of measurement microphone when the first 100 modes (the number of modes below Schroeder frequency) exist in the response of the kiosk. The part of this figure distinguished with narrow line depicts the SPL when summation (3) is computed over the first 1000 modes. As can be seen in Fig. 6, except for one position, the SPL has started to converge to its true value after the contribution of 100 modes. The case where the microphone is placed at $[0.5,0.5,1]$ corresponds to the nodal plane of most of the modes and hence the response of the kiosk has not been influenced by them. This will necessitate the contribution of much more modes for the SPL to converge to its true value as shown in Fig. 6.

\subsection{Parent selection}

Parent selection is done using proportionate selection with replacement, i.e. the probability of selecting an individual is proportional to its fitness with the following relation

$$
P_{s}(i)=\frac{f_{i}(t)}{\sum_{j=1}^{n_{p o p}} f_{j}(t)}=\frac{1}{N} \frac{f_{i}(t)}{\bar{f}(t)}
$$

where $f_{i}(t)$ is the fitness of $i^{t h}$ individual in the generation $t$ and $N$ is the number of populations in each generation. This selection mechanism is simulated with roulette-wheel method. Since some of the fitness may be negative (acoustic potential energy is increased in enclosure) they are added with their minimum to make all of them positive. This kind of selection may cause wandering the algorithm at final stages, because of no selective pressure on individuals and motivate the algorithm to converge before getting mature enough. This problem is alleviated with forced mutation discussed in the next part.

\subsection{Genetic operation and replacement}

To produce the next generation from the selected parents, a uniform crossover is performed on individuals. Also each individual with a low probability may experience mutation. To keep the diversity of population large enough, in spite of this probabilistic mutation the populations go under forced mutation. This operation assures that if the new offspring is equal to one of the other chromosomes in the same generation, one of its bits will change randomly until a new offspring different from other individuals is generated. Besides, all individuals of old generation are replaced totally with a new generation, and only the best individual in each generation is transmitted directly to the next generation (elitism). The flowchart of the proposed algorithm is depicted in Fig. 7. Since the optimal placement procedure is performed offline, and hence the convergence time is not limited, the terminating condition is when it is sensed that the algorithm will not be able to converge to a better solution.

\subsection{Tuning the genetic algorithm}

In order to obtain the proper performance of the genetic algorithm for the problem in hand, its parameters such as coding scheme, crossover probability, mutation probability, and initial population size are tuned with several simulations. In fact these parameters control the performance of the genetic algorithm by keeping a trade-off between the selective pressure of individuals with higher fitness and diversity of the populations. For each of the coding schemes (Integer coding and MVBS coding) the algorithm is executed several times. In each run, the position of the 
Table 1

Parameters of the genetic algorithm selected for comparison

\begin{tabular}{lc}
\hline Parameter & Value \\
\hline Cross over rate $(\mathrm{Pc})$ & $0.6,0.8,1$ \\
Mutation rate $(\mathrm{Pm})$ & 0.05 \\
Parent selection & Proportional \\
Crossover type & Uniform \\
Population size & 70 \\
Primary source location & {$[1,0.925,1.925]$} \\
Frequency & $50-300 \mathrm{~Hz}$ \\
\hline
\end{tabular}
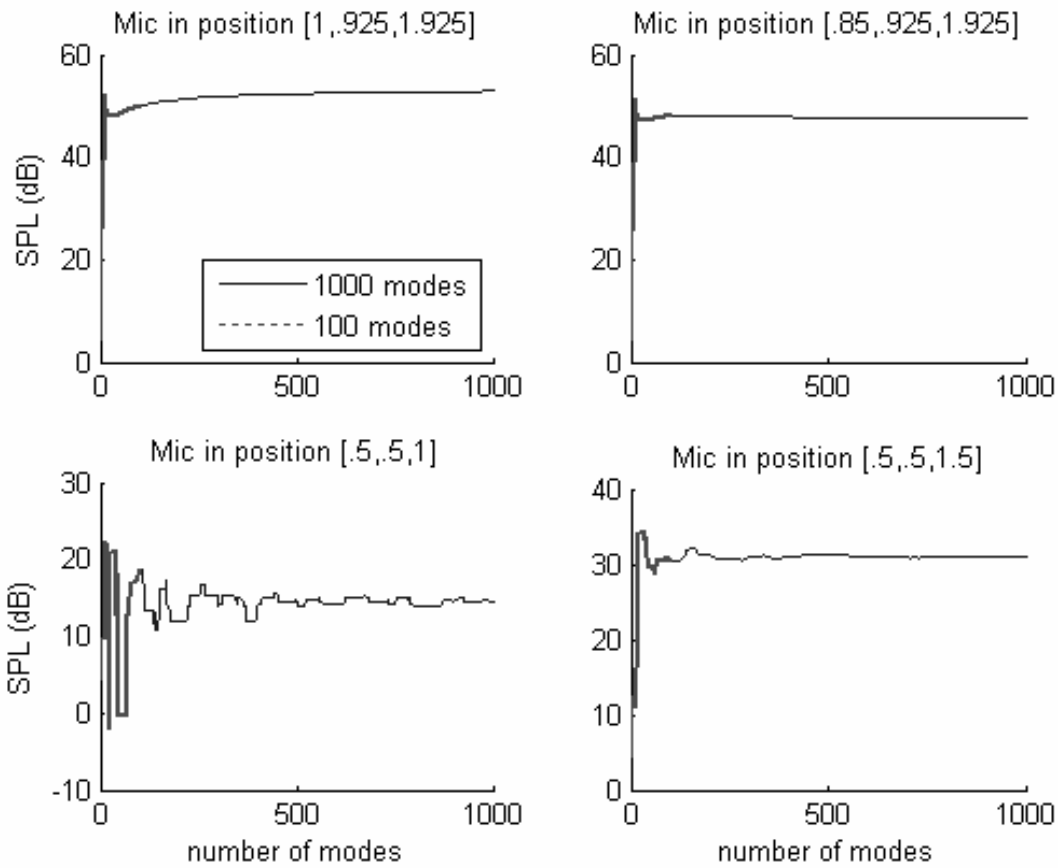

Fig. 6. Calculation of sound pressure level at $300 \mathrm{~Hz}$ in terms of increasing number of modes.

primary source is assumed to be fixed, and the frequency range where the performance index is minimized is 50 to $300 \mathrm{~Hz}$. Other parameters selected for comparison and tuning of the genetic algorithm are shown in Table 1.

For each of the MVBS coding, Integer coding and random search, the algorithm is executed 10 times with different crossover probabilities of $0.6,0.8$, and 1 as shown in Table 1 . The mean of the learning curve for example for Pc $=1$ and different coding schemes is shown in Fig. 8. Also the average of learning curves for one of the coding methods and different crossover probabilities are compared. Figure 9 shows the average of learning curves for Integer coding and different crossover probabilities. Since the genetic algorithms are executed many times and the final achievable reduction recorded by each run was a quantity near $30 \mathrm{~dB}$, it can be argued that the learning curves shown in Figs 8 and 9 have reached near the optimum positions and hence the cycle of genetic algorithm is stopped.

These simulation shows that in the MVBS coding, because of more diversity of populations at the beginning of the algorithm, it has faster convergence rate than Integer coding. However, after maturation of the generations, its search towards the higher-fitness individuals has stopped. On the other hand, Integer coding behaves in a different manner. In this case, the population diversity at the start of the algorithm is low and hence the algorithm converges with a lower rate than MVBS coding. However, after searching some primary generations, its population diversity is increased and the algorithm has the capability to find individuals with higher fitness than the algorithm using MVBS coding. The average of fitness for 10 runs of each case is summarized in Table 2. 


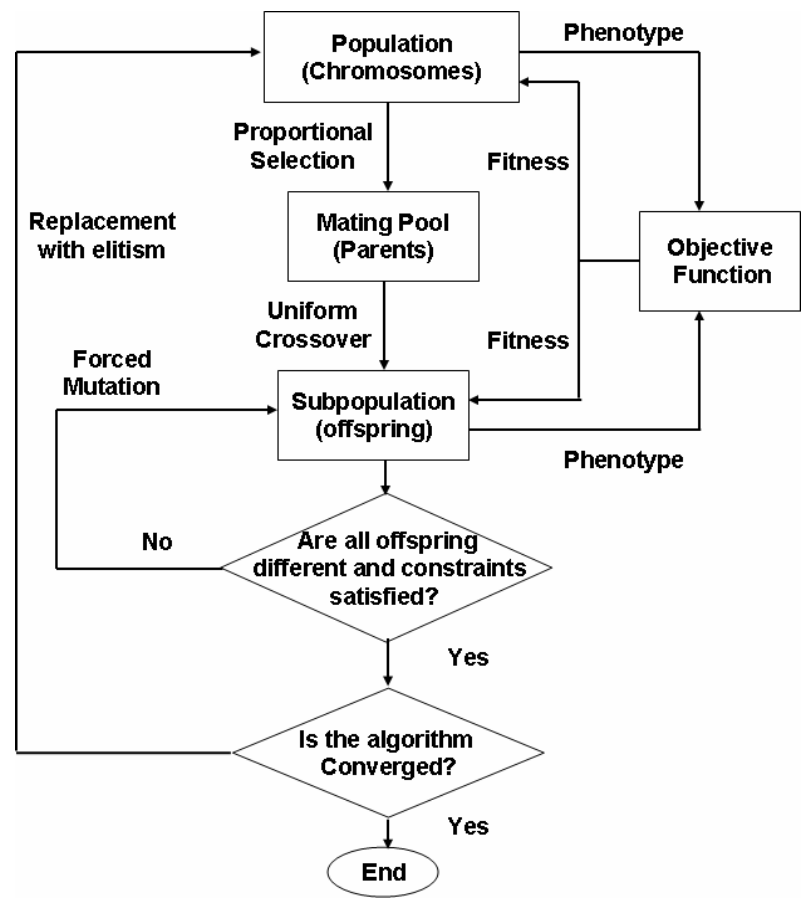

Fig. 7. Flowchart of the proposed genetic algorithm.

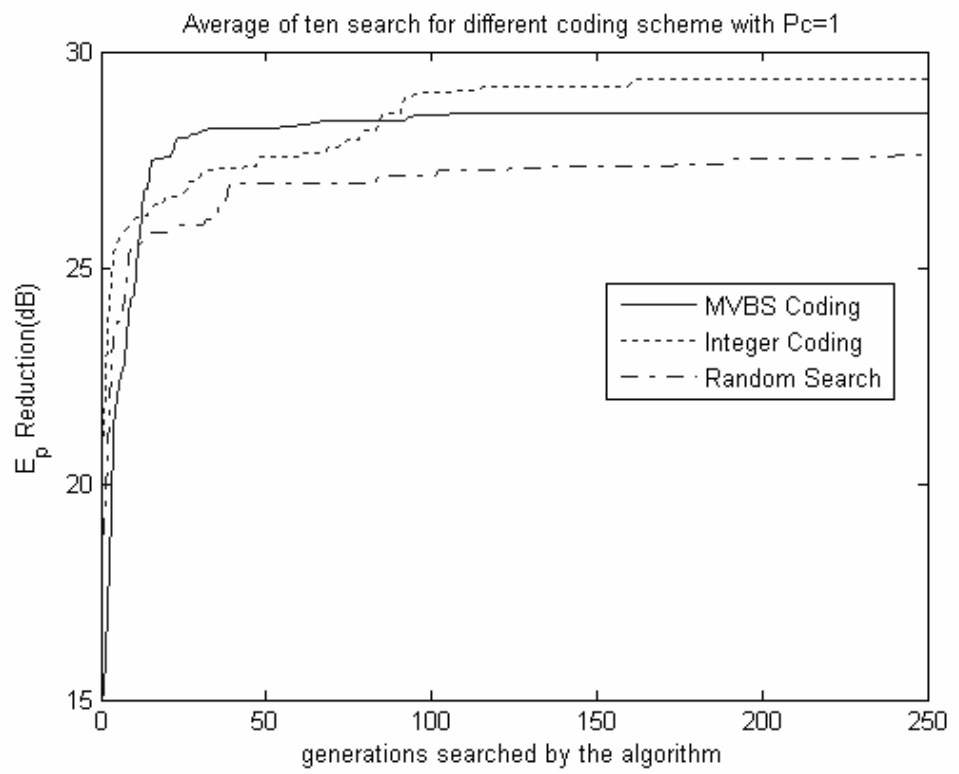

Fig. 8. Learning curve of the genetic algorithms with different coding schemes for $P c=1$.

As can be seen in Table 2, the best result is obtained with Integer coding and a crossover probability of 0.8 , and so these parameters are set for the rest of the algorithm. To investigate the effect of initial population size in finding solutions with higher fitness, an algorithm with MVBS coding and $\mathrm{Pc}=0.8$ for different initial population sizes of 20,50 , and 70 is executed.

The results after 10 runs and averaging the learning curves are shown in Fig. 10. As a result of these comparisons, 
Table 2

Evaluated fitness for different coding schemes and different crossover probabilities

\begin{tabular}{lccccccccc}
\hline & \multicolumn{3}{c}{ Max } & \multicolumn{4}{c}{ Min } & \multicolumn{4}{c}{ Average } \\
Pc & 1 & 0.8 & 0.6 & 1 & 0.8 & 0.6 & 1 & 0.8 & 0.6 \\
\hline MVBS Coding & 31.2466 & 33.3018 & 33.3020 & 27.7609 & 28.4539 & 291435 & 29.0514 & 29.6116 & 30.1697 \\
Integer Coding & 33.3020 & 30.3025 & 30.3402 & 27.8703 & 24.1794 & 26.8523 & 29.5939 & 31.6778 & 28.5554 \\
Random Search & 29.8687 & 29.8687 & 29.8687 & 27.0122 & 27.0122 & 27.0122 & 27.6823 & 27.6823 & 27.6823 \\
\hline
\end{tabular}

Table 3

The parameters of the best genetic algorithm proposed for optimization

\begin{tabular}{lc}
\hline Parameter & Value \\
\hline Crossover rate & 0.8 \\
Mutation rate & 0.05 \\
Population size & 70 \\
Parent selection & Proportional \\
Crossover type & Uniform \\
Coding scheme & Integer coding \\
Fitness & Mean of Ep in 50-300 Hz \\
\hline
\end{tabular}

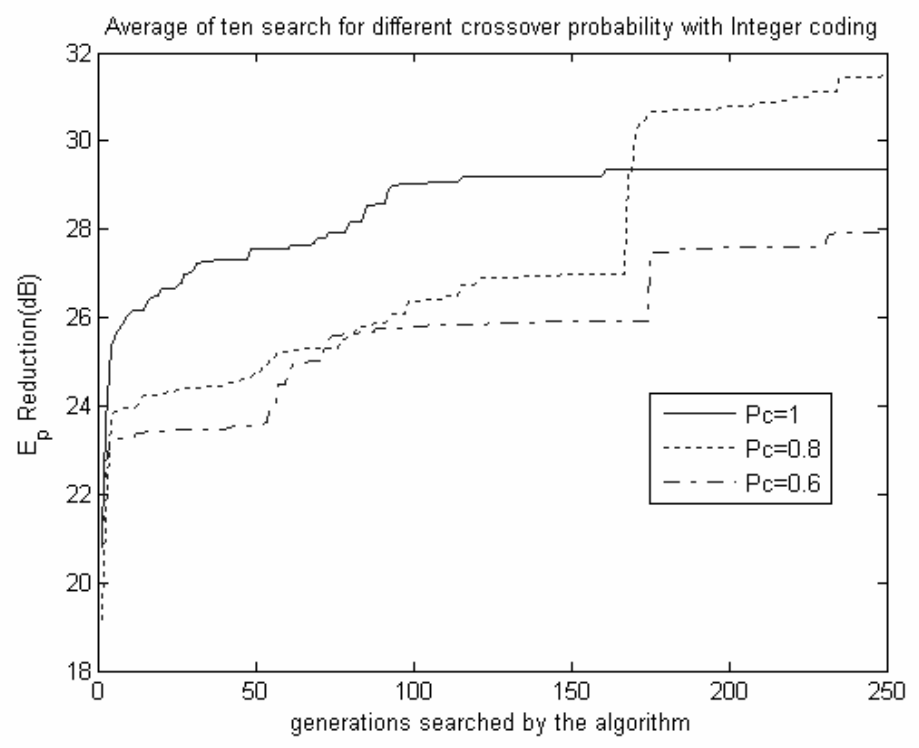

Fig. 9. Learning curve of the genetic algorithms for Integer coding and different crossover probabilities.

the parameters of the best genetic algorithm proposed for this optimization problem is shown in Table 3.

\section{Evaluation of the optimization results}

By executing the algorithm several times, different arrangements for loudspeakers and microphones will be generated near the optimal solution. Among these runs, one of the best results is selected. The coded outputs of the algorithm and their equivalent coordinates in the selected coordinate system are shown in the left column of Table 4. In the right column a sample of the output of the algorithm for MVBS coding is listed.

The optimal positions of loudspeakers and microphones listed in the left column of Table 4 are illustrated in Fig. 11. In order to evaluate these results, an ANC system was simulated, considering the locations proposed by the genetic algorithm for loudspeakers and microphones. 
Table 4

outputs from algorithm and their equivalent coordinates

\begin{tabular}{ccccc}
\hline Spk./Mic. & Code & Equivalent position & Code & Equivalent position \\
\hline S1 & 3584 & $0.8117,0.9250,1.9250$ & 111010001000 & $0.6983,0.9250,1.9250$ \\
S2 & 3328 & $0.7550,0.9250,1.9250$ & 101110001000 & $0.8117,0.9250,1.9250$ \\
M1 & 4014 & $0.9250,0.6417,1.6783$ & 101001011101 & $0.7550,0.4150,1.1850$ \\
M2 & 242 & $0.0750,0.9250,0.1983$ & 010000011011 & $0.4717,0.1317,1.6783$ \\
M3 & 2424 & $0.5850,0.4717,0.9383$ & 011100001011 & $0.3583,0.0750,1.6783$ \\
M4 & 1026 & $0.3017,0.0750,0.1983$ & 100101001000 & $0.8683,0.4717,1.9250$ \\
& Fitness $(\mathrm{dB})$ & 33.3017 & Fitness $(\mathrm{dB})$ & 33.3008 \\
\hline
\end{tabular}

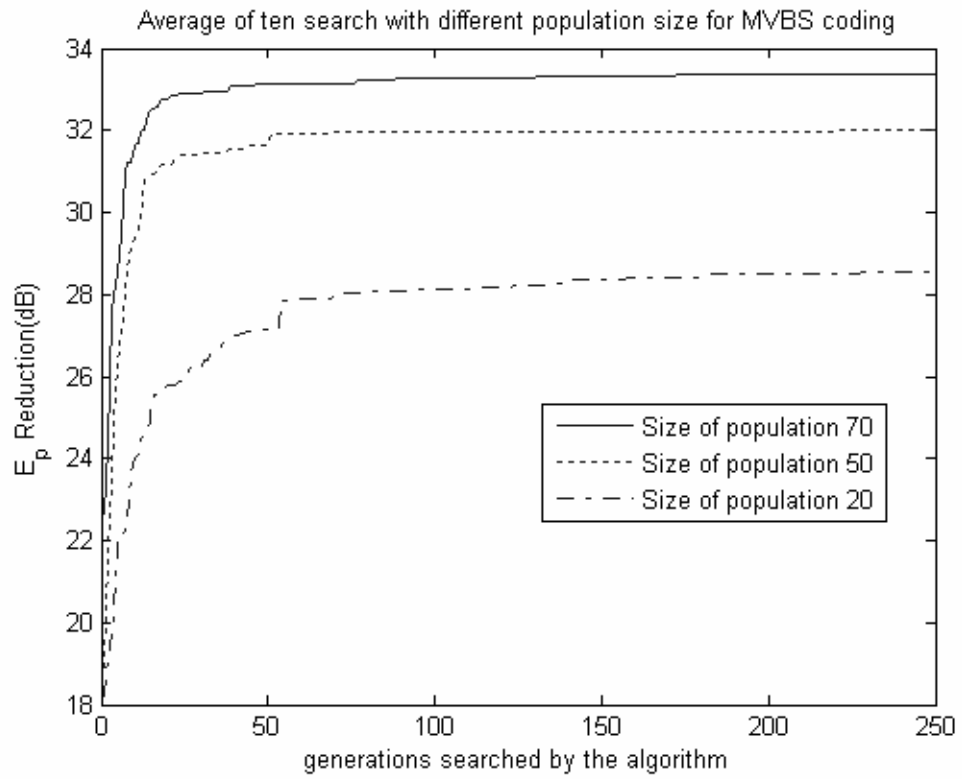

Fig. 10. Learning curve of genetic algorithm with MVBS coding and different population sizes.

Figure 12 depicts the acoustic potential energy level in the specified frequency range before and after the ANC is applied. As can be obviously observed in this figure, the peaks of resonant frequencies in enclosure have been flattened, and hence for a broadband incident disturbance whose energy is concentrated in the desired frequency range the potential energy has been reduced substantially. In fact, the obtained reductions determine an upper bound for the real ANC system which may be implemented with time domain algorithms such as multi-channel FxLMS. The acoustic pressure pattern at different levels, especially at the height of $1.5 \mathrm{~m}$ and $1.65 \mathrm{~m}$ in the enclosure is shown before and after running the ANC system in Figs 13 and 14. These heights correspond to the location of average ears level for women and men standing in the kiosk respectively. In this simulation, reduction of the sound pressure level at this height verifies the performance of the control system simulation based on the locations found by the genetic algorithm.

To check the robustness of the obtained performance regarding eventual uncertainties which may exist in real applications, three kinds of uncertainties are considered:

1-uncertainty in the position of primary loudspeaker

2-uncertainty in the position of secondary loudspeaker

3-uncertainty in the position of microphones

Robustness of the performance with respect to uncertainties in the positions of loudspeakers is very important. This is due to practical limitations that may exist in their installation. What is meant by robustness analysis is to examine how the average of acoustic potential energy reduction in the enclosure is influenced by changes in the position of loudspeakers and microphones. As a matter of fact, if the degradation in the performance of the control 


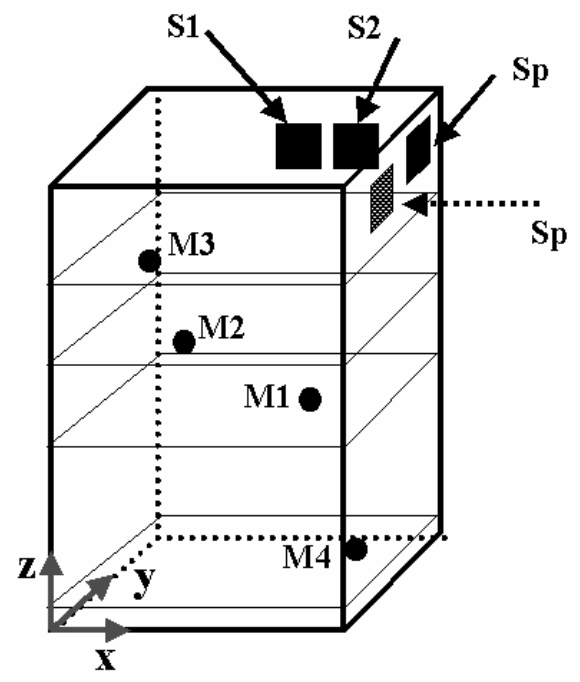

Fig. 11. Optimal arrangement of loudspeakers and microphones in the kiosk.

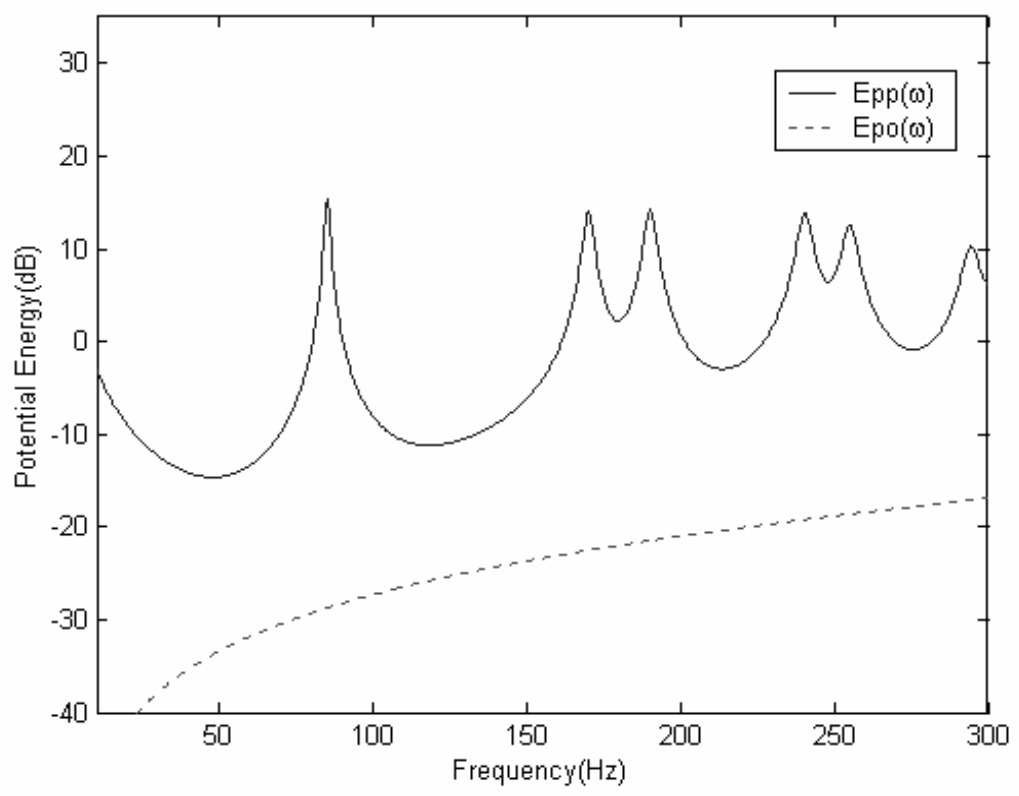

Fig. 12. The performance of the ANC system in frequency domain with the arrangement shown in Fig. 11.

system remains small against small perturbations in the positions of loudspeakers and microphones, it can be said that the robustness is good; otherwise the optimal configuration will show poor robustness. In all cases random perturbations in $x, y$, and $z$ directions with magnitudes of $0.005,0.05,0.2$ and 0.5 are added to the optimal coordinates of loudspeakers and microphones shown in Fig. 11. Then by calculating the deviation of average acoustic potential energy reduction from its optimal value and taking its mean value for ten different realizations of perturbations, the sensitivity of the performance index with respect to these perturbations is computed. The results are summarized in Table 5. From the analysis results of this table the following results can be deduced clearly: 1- The robustness of the optimal configuration with respect to deviations in the microphone positions is much higher than that of 

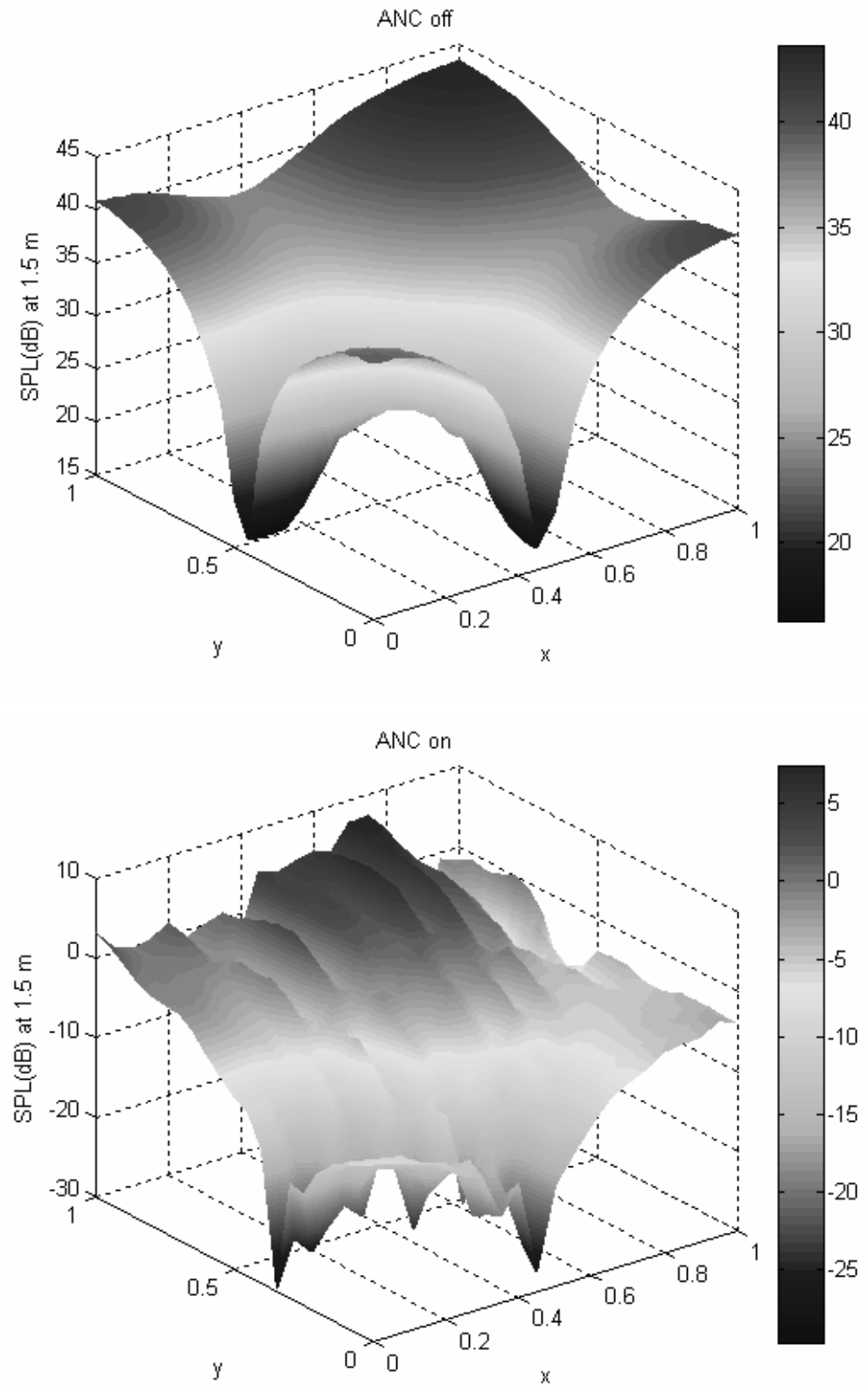

Fig. 13. Distribution of acoustic pressure at the height of $1.5 \mathrm{~m}$.

loudspeakers. 2- By increasing the magnitude of perturbations of secondary loudspeakers from their optimal values, the deviation from optimal performance is increased with reducing sensitivity. 3- Speaker S1 is more responsible than speaker S2 in achieving the optimal performance and hence its deviation degrades the performance more. 4The performance of optimal configuration is also sensitive to the position of the primary speaker, but not as much as it is to S1. The acoustic potential energy of enclosure for one perturbation in Sp, for example, is plotted in Fig. 15. Figure 16 shows the acoustic potential energy of enclosure when the positions of S1 and S2 are perturbed. In this case the microphones are assumed to be placed in optimal positions. And finally Fig. 17 shows the performance of the ANC system when all four microphones are perturbed according to Table 5. It can be seen that these figures also confirm the results of Table 5 . 

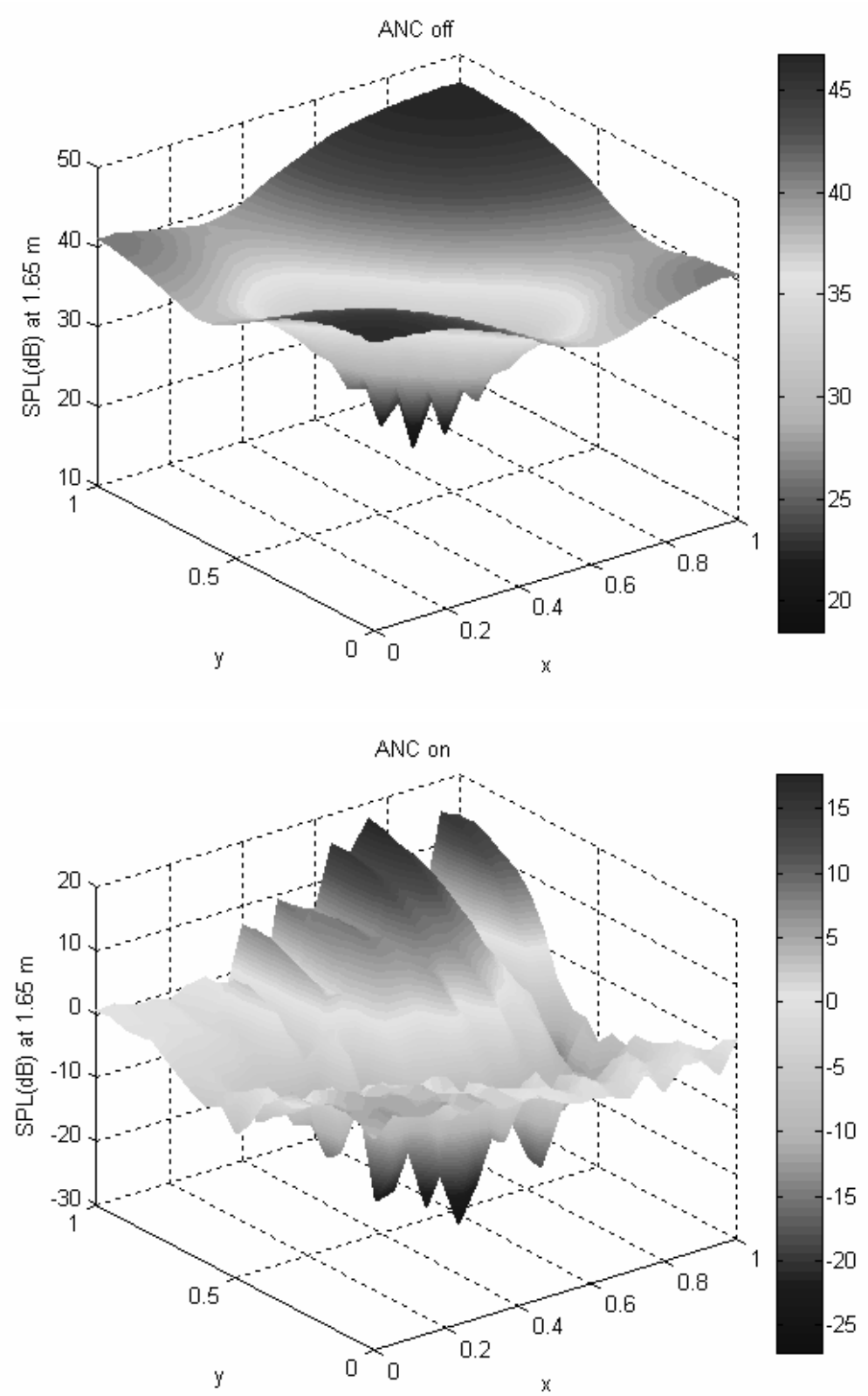

Fig. 14. Distribution of acoustic pressure at the height of $1.65 \mathrm{~m}$.

In order to have an idea of what the suitable size of the control system is (number of loudspeakers and microphones) for global reduction of noise in the kiosk, the genetic algorithm is executed from one up to five secondary speakers, assuming exact knowledge of modal characteristics of the kiosk to avoid using any microphones in optimization procedure. The maximum reduction of acoustic potential energy for each case is obtained and the results are summarized in Table 6. Since the microphones are used to estimate the acoustic potential energy of the enclosure, increasing their number will theoretically result in a better approximation. Nevertheless, to keep the size of control system and hence its complexity in a reasonable range the number of microphones must be the minimum number where the performance of the system is not degraded. It can be seen that the best result is obtained with four secondary loudspeakers. However the best size of the control system may be limited by the available hardware used 
Table 5

Robustness analysis of the performance index with respect to positions of speakers and microphones

\begin{tabular}{cccc}
\hline & $\begin{array}{c}\text { Perturbation } \\
\text { mag. }(\mathrm{m})\end{array}$ & $\begin{array}{c}\text { Deviation in } \\
\text { average APR }(\mathrm{dB})\end{array}$ & Sensitivity \\
\hline $\mathrm{Sp}$ & 0.005 & 0.2056 & 4.0730 \\
& 0.05 & 0.4265 & 0.8449 \\
& 0.2 & 6.3815 & 3.1603 \\
$\mathrm{~S} 1$ & 0.5 & 14.7156 & 2.9150 \\
& 0.005 & 0.2507 & 4.8105 \\
& 0.05 & 4.9708 & 9.5398 \\
S2 & 0.2 & 12.6108 & 6.0506 \\
& 0.5 & 13.1160 & 2.5172 \\
& 0.005 & 0.3304 & 6.2866 \\
S1, S2 & 0.05 & 1.5884 & 3.0225 \\
& 0.2 & 4.6090 & 2.1925 \\
& 0.5 & 5.0036 & 0.9521 \\
& 0.005 & 0.3538 & 6.7329 \\
& 0.05 & 3.3751 & 6.4220 \\
& 0.2 & 17.7599 & 8.4483 \\
M1,M2,M3,M4 & 0.5 & 23.4938 & 4.4703 \\
& 0.005 & 0.2317 & 4.4078 \\
& 0.05 & 0.1739 & 0.3553 \\
& 0.2 & 0.0542 & 0.0277 \\
& 0.5 & 0.1199 & 0.0245 \\
\hline
\end{tabular}

Table 6

The optimal fitness by increasing the number of secondary speakers

\begin{tabular}{cc}
\hline $\begin{array}{c}\text { No. of } \\
\text { speakers }\end{array}$ & $\begin{array}{c}\text { Max. reduction achieved } \\
\text { by algorithm }(\mathrm{dB})\end{array}$ \\
\hline 1 & 19.1819 \\
2 & 29.1053 \\
3 & 32.9162 \\
4 & 34.1801 \\
5 & 33.1791 \\
\hline
\end{tabular}

for implementation.

\section{Conclusions}

The importance of suitable locations for error microphones and control speakers in the stability and maximum achievable global reduction of acoustic pressure, demands a method for their optimal placement in a multi-channel ANC system. Since in systems with large dimensions, the number of possible locations for placement of these sensors and actuators are uncountable, the assessment of all configurations is impossible, and hence a guided random search method such as a genetic algorithm is very useful for this purpose. By selecting a proper cost function to represent the acoustic potential energy in an enclosure in the interested frequency range, the proposed genetic algorithm can find the best locations for loudspeakers and microphones in a specific routine by evaluating the fitness of individual placement. In fact, this algorithm by considering the defined constraints, tries to find the global maximum of the defined cost function. Results from simulation of acoustic pressure and also acoustic potential energy in the enclosure verify the efficient placement by the proposed genetic algorithm. Since many aspects in the acoustics of the kiosk is not correctly previewed by the modal series model, it usually does not match closely experimental data. In order for the results to be applicable to a real kiosk, it is necessary to compare the frequency response function arising from the numerical model with that measured from experimental data. The robustness of the performance of the system with respect to changes in the obtained positions for primary and secondary loudspeakers and also microphones is shown by several simulations. This robustness analysis is important because of limitations that may exist in practical implementations of the control system. 


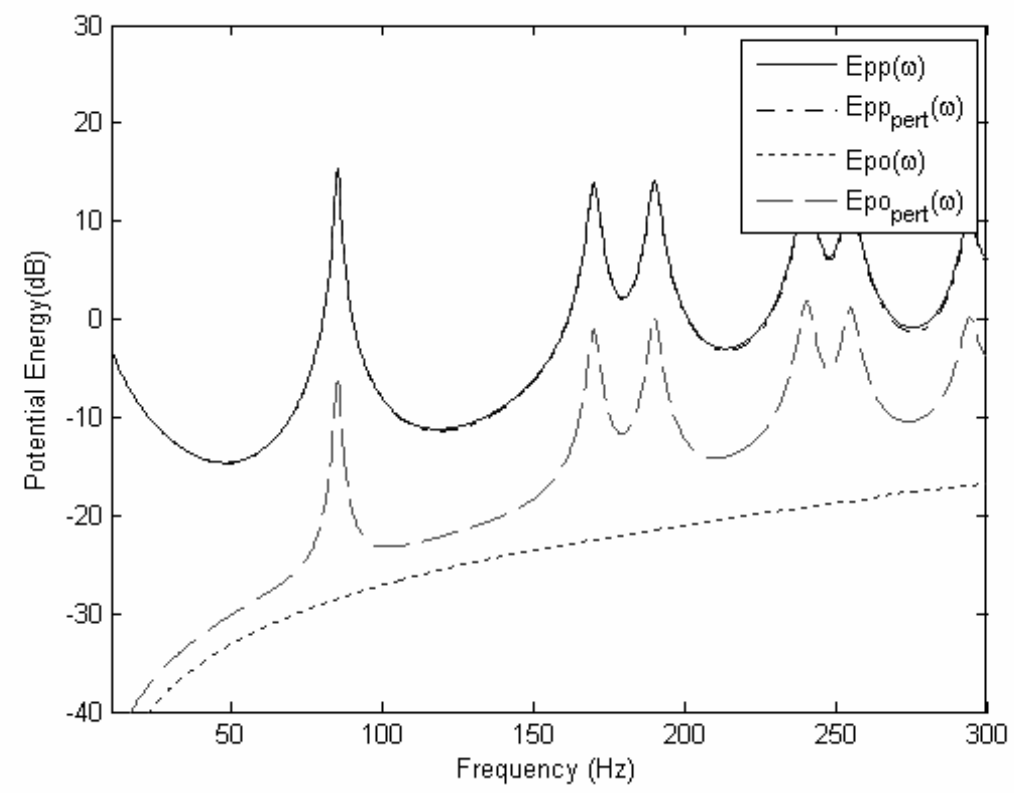

Fig. 15. The performance of the ANC system when the position of primary speaker is perturbed.

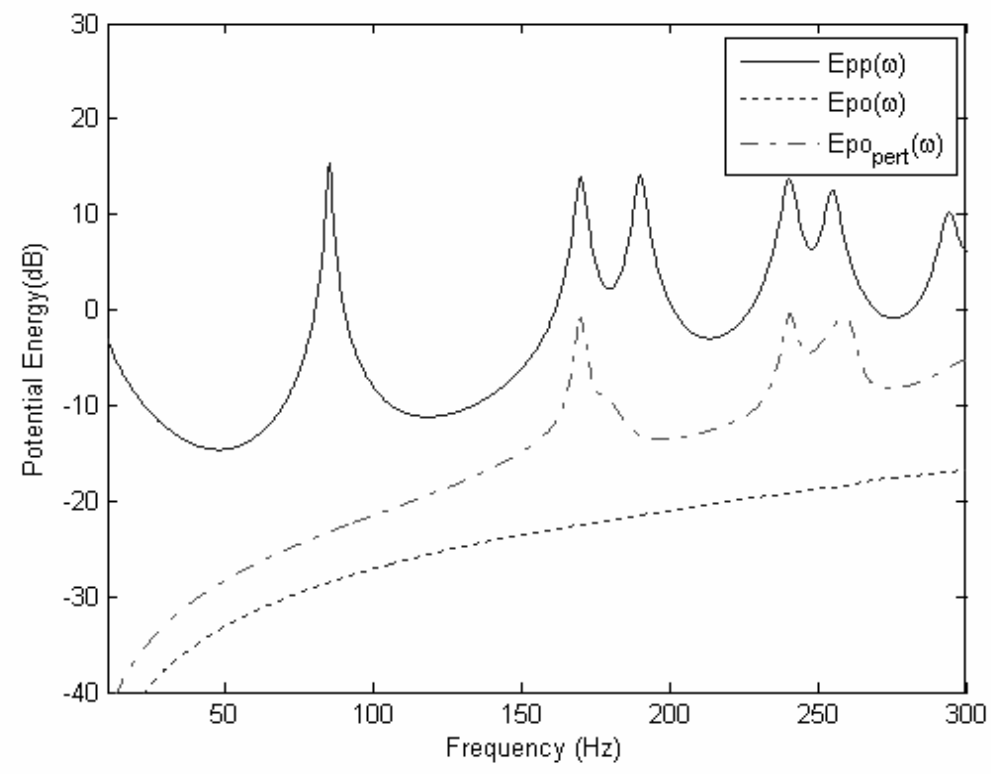

Fig. 16. The performance of the ANC system when the positions of both secondary speakers are perturbed.

\section{APPENDIX A}

The mode shape and its amplitude can be calculated as follows

$$
\psi_{n}(\mathbf{x})=\sqrt{\varepsilon_{n_{1}} \varepsilon_{n_{2}} \varepsilon_{n_{3}}} \cos \left(\frac{n_{1} \pi x}{L_{1}}\right) \cos \left(\frac{n_{2} \pi y}{L_{2}}\right) \cos \left(\frac{n_{3} \pi z}{L_{3}}\right)
$$




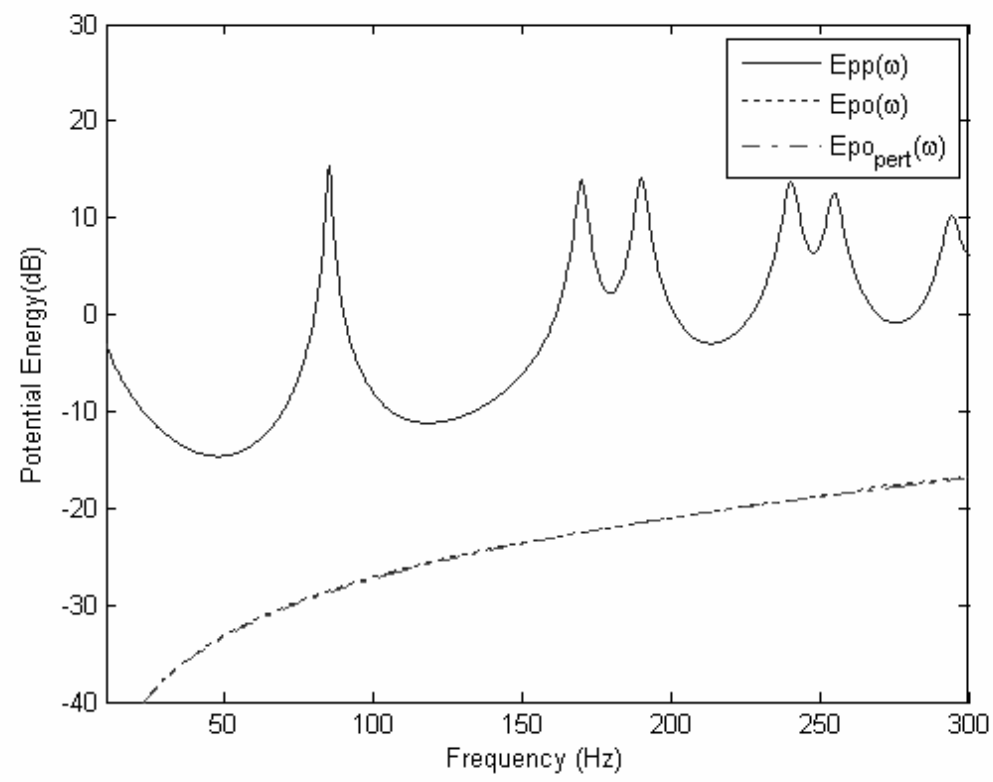

Fig. 17. The performance of the ANC system when the positions of microphones are perturbed.

$$
a_{n}(\omega)=\frac{\rho_{0} c_{0}^{2}}{V} A_{n}(\omega) \int_{V} G(\mathbf{x}, \omega) \psi_{n}(\mathbf{x}) d V,
$$

where $L_{1}, L_{2}$ and $L_{3}$ are the dimensions of enclosure, and $n_{1}, n_{2}$ and $n_{3}$ are natural numbers specifying the mode order. Besides

$$
\varepsilon_{v}= \begin{cases}2 & \text { if } \quad v>0 \\ 1 & \text { if } \quad v=0\end{cases}
$$

and $A_{n}(\omega)$ is defined as

Considering the principle of superposition, the amplitude of each mode at a certain frequency can be expressed as $\mathbf{a}=\mathbf{a}_{p}+\mathbf{B q}_{s}$,

where for a distributed source with surface $S$, the element $a_{\mathrm{p} n}$ of vector $\mathbf{a}_{P}$ is the amplitude related to mode $n$, and the element $B_{n m}$ of matrix $\mathbf{B}$ are obtained from

$$
\begin{gathered}
a_{\mathbf{p}_{n}}(\omega)=\left(\rho_{0} c_{0}^{2} / V\right) A_{n}(\omega) V_{p}(\omega) \int_{\mathbf{S}_{p}} \psi_{n}(\mathbf{y}) d s \\
B_{n m}(\omega)=\left(\rho_{0} c_{0}^{2} / V\right) A_{n}(\omega)(1 / S) \int_{\mathbf{S}_{m}} \psi_{n}(\mathbf{y}) d s .
\end{gathered}
$$

Here $V_{p}(\omega)$ is the volume velocity per unit surface of the loudspeaker, and is assumed uniform on the entire speaker surface.

Acoustic pressure at $M$ discrete point of the enclosure can be written as

$$
\mathbf{p}=\Psi_{L}^{T} \mathbf{a},
$$

where $\Psi_{L}$ is an $M \times N$ matrix whose element $(m, n)$ is the value of mode shape of $n$th mode at point $m$. Replacing $\mathbf{a}$ in terms of $\mathbf{a}_{P}$ and $\mathbf{q}_{s}$ will yield 


$$
\mathbf{p}=\mathbf{p}_{p}+\mathbf{Z} \mathbf{q}_{s},
$$

where $\mathbf{p}_{p}=\Psi_{L}^{T} \mathbf{a}_{p}$ is the primary acoustic pressure and $\mathbf{Z}=\Psi_{L}^{T} \mathbf{B}$ the transfer function between secondary sources and microphones.

\section{References}

[1] K.H. Back and S.J. Elliott, Natural algorithms for choosing source locations in active noise control systems, Journal of Sound and Vibration 186(2) (1995), 245-267.

[2] A.J. Bullmore, P.A. Nelson, A.R.D. Curtis and S.J. Elliott, Active minimization of harmonic enclosed sound fields, Part II: A Computer Simulation, Journal of Sound and Vibration 117(1) (1987), 15-33.

[3] P. Defonseca, P. Sas and H. Brussel, Optimization methods for choosing sensor and actuator locations in an actively controlled double-panel partition, In Proceedings of the SPIE 3041 (1997), 124-135.

[4] Z.G. Diamantis, D.T. Tsahalis and I. Botchers, Optimization of an Active Noise Control System Inside an Aircraft, Based on the Simultaneous Optimal Positioning of Microphones and Speakers, with the Use of a Genetic Algorithm, Computational Optimization and Applications 23(1) (2002).

[5] S.J. Elliott, Signal Processing for Active Control, Pub. Academic Press 2001.

[6] D.E. Goldberg, Genetic algorithms in search optimization and machine learning, Addison-Wesley, 1989.

[7] C.H. Hansen, M.T. Simpson and B.S. Cazzolato, Genetic algorithms for active sound and vibration Control, Inter-Active 99, Web Forum held by the IEE Control System Theory and Design Committee (1999).

[8] C.H. Hansen, Understanding Active Noise Control, Pub. Brunner-Routledge, 2001.

[9] S. Laugesen and S.J. Elliott, Multichannel Active Control of Random Noise in a Small Reverberant Room, IEEE Transactions on Speech and Audio Processing 1(2) (1993), 241-249.

[10] H. Li, J. Chen, K. Chen and Y. Tan, Optimal Arrangement of Error Sensors in AANC System Based on the Genetic Algorithm, Proceedings of the 3rd IEEE world congress on Intelligent Control and Automation 1 (2000), 561-563.

[11] T. Martin and A. Roare, Active noise control of acoustic sources using spherical harmonic expansion and a genetic algorithm: simulation and experiment, Journal of Sound and Vibration 212 (1998), 511-523.

[12] A. Montazeri, J. Poshtan and M.H. Kahaei, Analysis of the Global Reduction of Broadband Noise in a Telephone Kiosk Using a MIMO Modal ANC System, International Journal of Engineering Science 45 (2007), 679-697.

[13] C.E. Ruckman and C.R. Fuller, Optimizing actuator locations in active noise control systems using subset selection, Journal of Sound and Vibration 186 (1995), 395-406.

[14] S.D. Snyder and C.H. Hansen, Using multiple regression to optimize active noise control system design, Journal of Sound and Vibration 148(3) (1991), 537-542.

[15] P.J. van Laarhoven and E.H. Aarts, Simulated Annealing: Theory and Applications, Kluwer Academic Publishers, 1992.

[16] E. Bonabeau, M. Dorigo and G. Theraulaz, Swarm Intelligence: from natural to artificial systems, Oxford University Press, 1999.

[17] D.C. Zimmerman, A Darwinian approach to the actuator number and placement problem with non-negligible actuator mass, Journal of Mechanical System and Signal Processing 7(4) (1993), 363-374.

[18] M.R. Schroeder, The Schroeder frequency revisited, Journal of Acoustic Society of America 99(5) (1996), 3240-3241. 

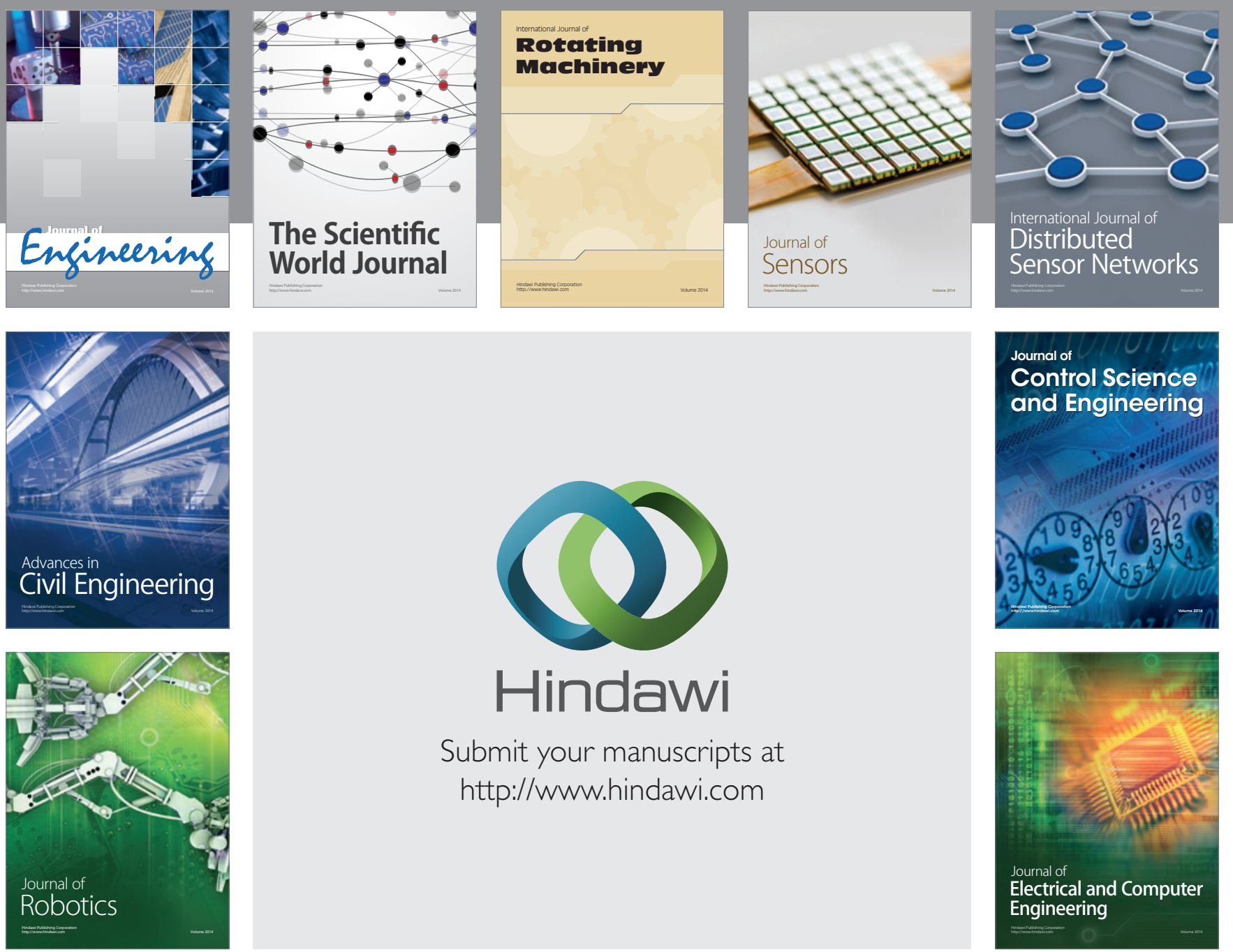

Submit your manuscripts at

http://www.hindawi.com
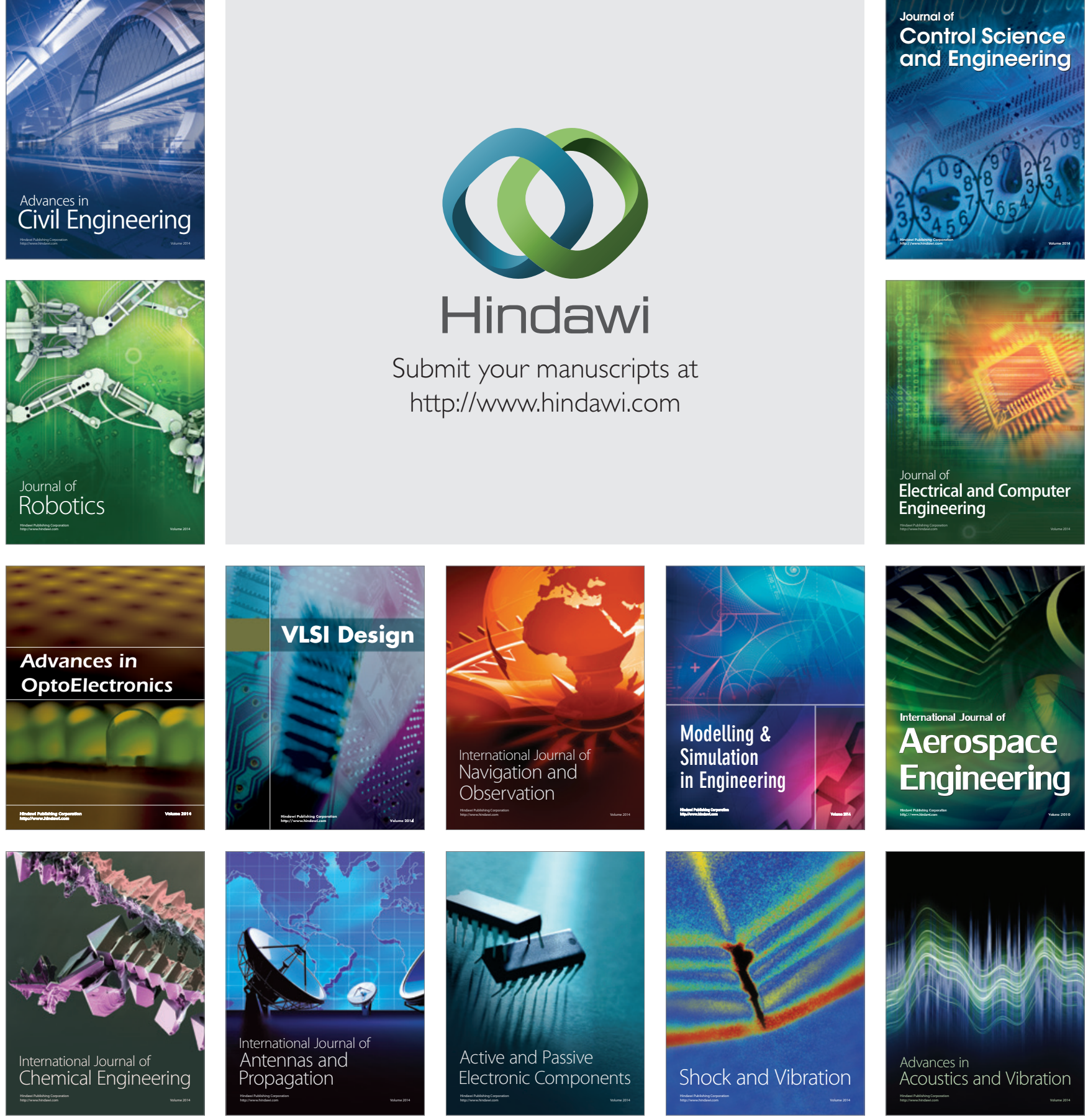